Supporting Information for:

\title{
Tuning the Surface Chemistry of Melanin-Mimetic Polydopamine Nanoparticles Drastically Enhances their Accumulation into Excised Human Skin
}

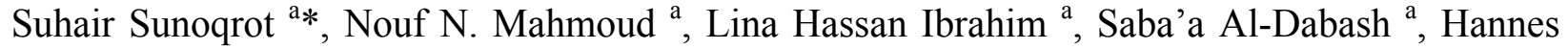
Raschke $^{\mathrm{b}}$, and Roland Hergenröder ${ }^{\mathrm{b}}$

${ }^{a}$ Department of Pharmacy, Faculty of Pharmacy, Al-Zaytoonah University of Jordan, Amman 11733, Jordan

${ }^{b}$ Leibniz-Institut fuer Analytische Wissenschaften - ISAS - e.V., Bunsen-Kirchhoff Str. 11, D44139 Dortmund, Germany

*Corresponding Author:

Suhair Sunoqrot, $\mathrm{PhD}$

Associate Professor of Pharmaceutics

Department of Pharmacy

Faculty of Pharmacy

Al-Zaytoonah University of Jordan

P.O. Box 130, Amman 11733, Jordan

Phone: +962-6-4291511 Ext. 197

Fax: +962-6-4291432

Email: suhair.sunoqrot@,zuj.edu.jo

No. of pages: 12

No. of figures: 10 


\section{Characterization of PDA NPs}

Particle size and zeta potential of the NPs prepared in this study were measured by DLS using a Nicomp Nano Z3000 instrument (Particle Sizing Systems, Santa Barbara, CA, USA). Freshly prepared NPs diluted in ultrapure water were used for the analysis. Each measurement was performed in triplicate. For TEM imaging, $10 \mu \mathrm{L}$ of NP aqueous dispersions was placed on 300mesh Formvar-coated copper grids (Electron Microscopy Sciences, Hatfield, PA, USA) for 1 min. After wicking excess liquid with filter paper, grids were imaged using a Morgagni 268 TEM (FEI, Netherlands) at $60 \mathrm{kV}$ accelerating voltage. For FT-IR analysis, NPs were freezedried (FreeZone 4.5 L benchtop freeze dryer, Labconco Corporation, Kansas City, MO, USA) and prepared as KBr discs. Spectra were recorded using a Shimadzu IR Affinity-1 spectrometer (Kyoto, Japan).

XPS was employed to verify successful immobilization of PEG ligands on PDA NPs. NP samples $(10 \mu \mathrm{L})$ were drop-casted onto pre-cleaned gold-coated silicon wafers $(18 \times 20 \mathrm{~mm})$ and left to dry overnight. Measurements were carried out using a near-ambient pressure XPS instrument (PHOIBOS 150 hemispherical analyzer, SPECS Surface Nano Analysis GmbH, Berlin, Germany) utilizing a monochromated Al Ka x-ray source (SPECS $\mu$-Focus 600) with a photon energy of $1,486.71$ operating at $14 \mathrm{kV}, 9 \mathrm{~mA}$, and $55 \circ \mathrm{C}$ take-off angle. The beam was focused to a spot size of ca. $600 \mu \mathrm{m}$ on the sample surface, and three spots were scanned per sample. Survey scans were performed between $0-1200 \mathrm{eV}$ and high-resolution scans were performed for the $\mathrm{C} 1 \mathrm{~s}$ and $\mathrm{O} 1 \mathrm{~s}$ regions. Data analysis and peak deconvolution was conducted using Igor Pro version 7.08. 
A
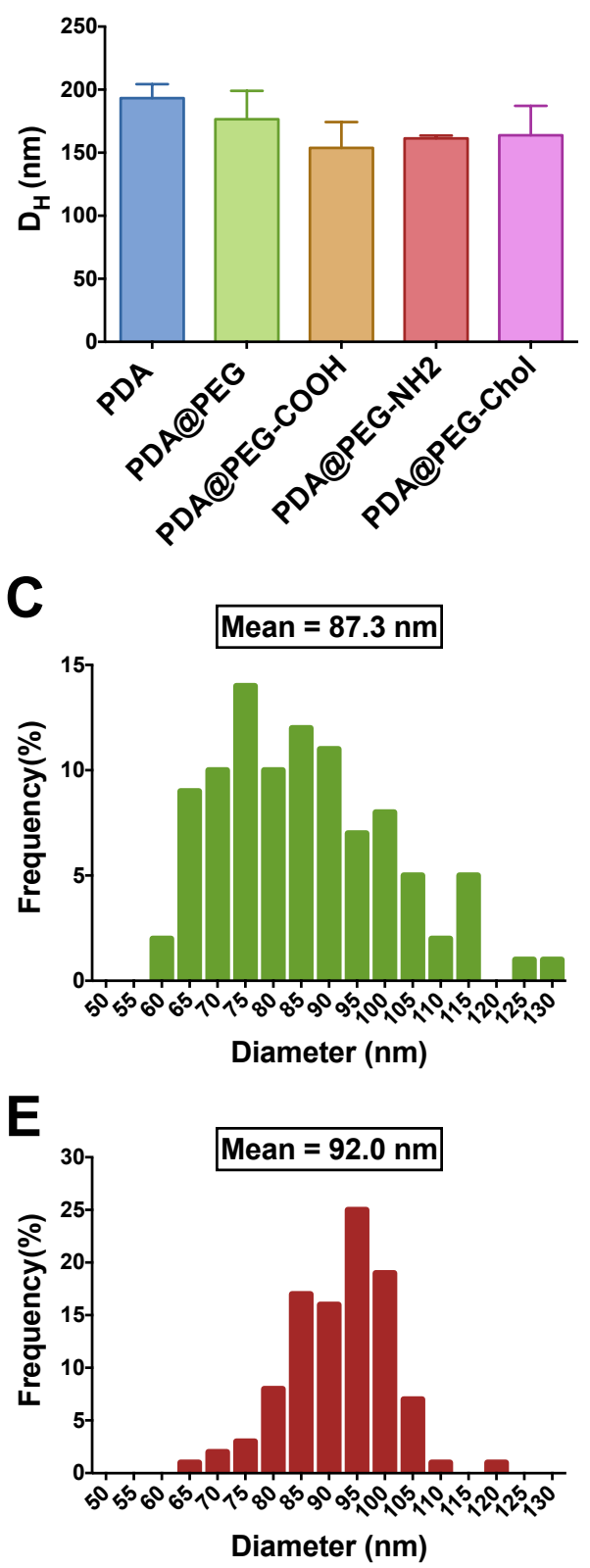

B

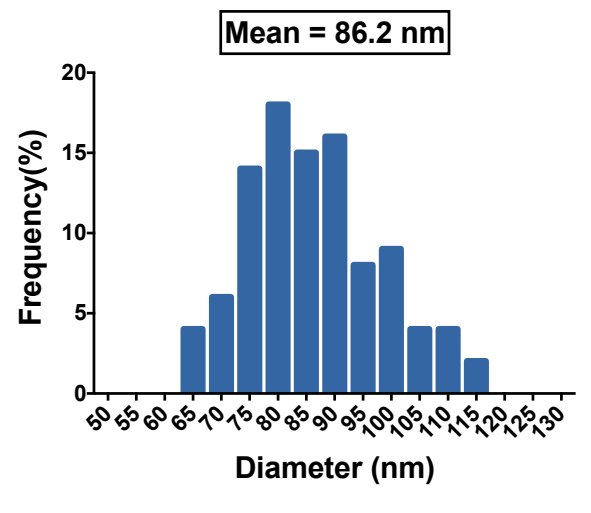

D

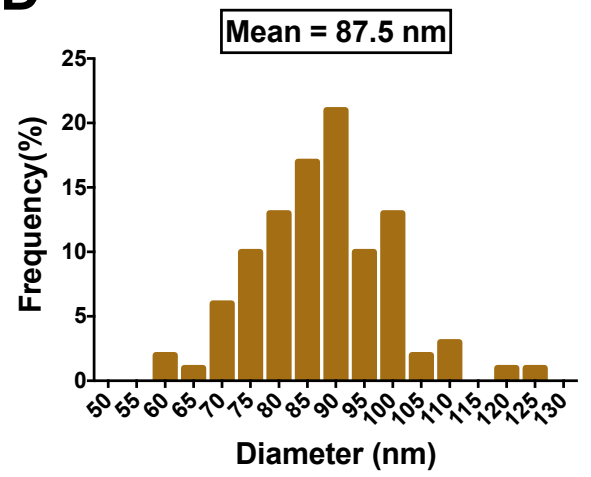

F

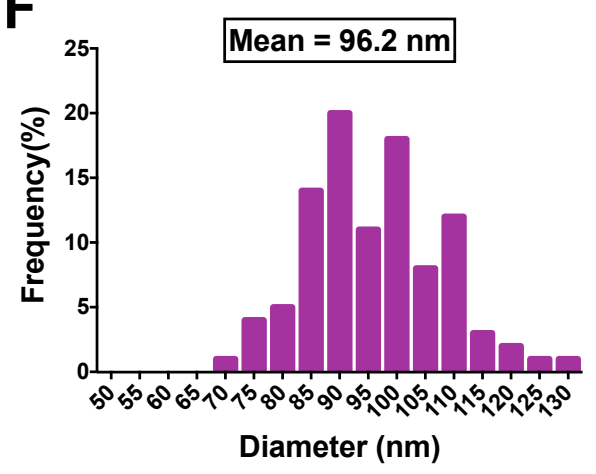

Figure S1. (A) Hydrodynamic diameters $\left(\mathrm{D}_{\mathrm{H}}\right)$ of PDA NPs before and after surface modification with various PEG ligands as measured by DLS. No statistical difference was observed between NP diameters based on one-way ANOVA followed by Tukey's post-hoc test. Results are expressed as mean \pm SD from three different batches of NPs; (B) - (F) Frequency distribution histograms of NP diameters for unmodified PDA (B), PDA@PEG (C), PDA@PEG-COOH (D), PDA@PEG-NH 2 (E), and PDA@PEG-Chol (F) NPs obtained by measuring the diameter of ca. 100 particles from TEM images using ImageJ (version $1.51 \mathrm{~m} 9$, NIH, Bethesda, MD, USA). 
A

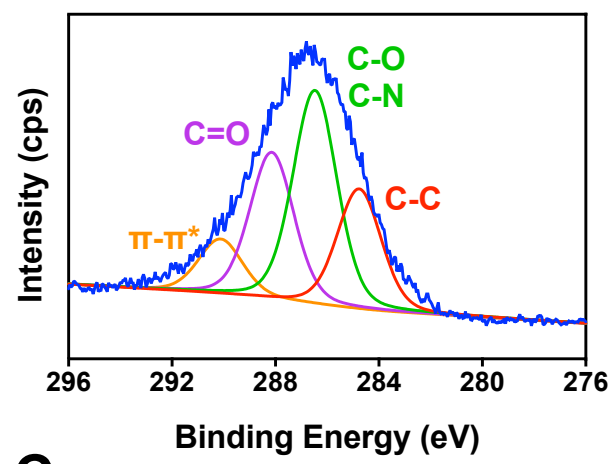

C

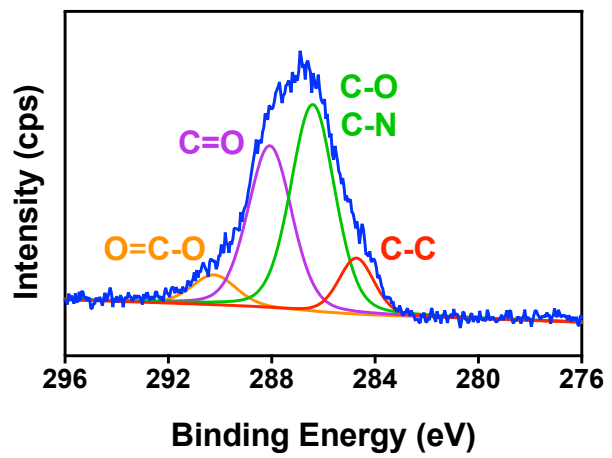

B

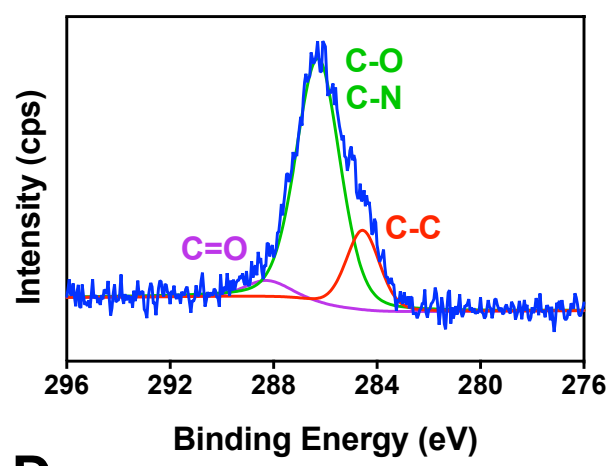

D

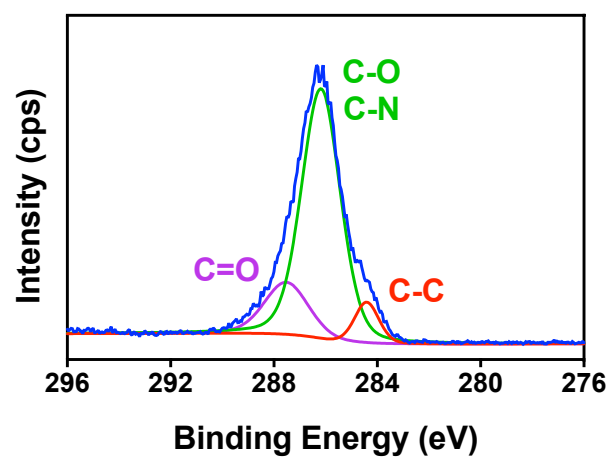

E

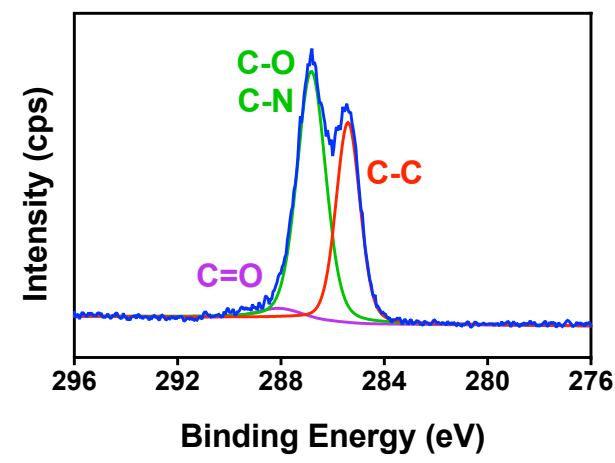

Figure S2. Deconvoluted XPS high-resolution spectra of $\mathrm{C} 1 \mathrm{~s}$ regions of (A) unmodified PDA, (B) PDA@PEG, (C) PDA@PEG-COOH, (D) PDA@PEG-NH 2 , and (E) PDA@PEG-Chol NPs with peak assignments inferred from literature. The increase in $\mathrm{C}-\mathrm{O}$ components observed in PEGylated NPs (B - E) compared to unmodified PDA NPs (A) is attributed to immobilization of $\mathrm{PEG}$ ligands on the surface of PDA NPs. The appearance of $\mathrm{O}=\mathrm{C}-\mathrm{O}$ components in $(\mathrm{C})$ and the increase in $\mathrm{C}-\mathrm{C}$ components in (E) correspond to the presence of $\mathrm{PEG}-\mathrm{COOH}$ and $\mathrm{PEG}-\mathrm{Chol}$, respectively. 

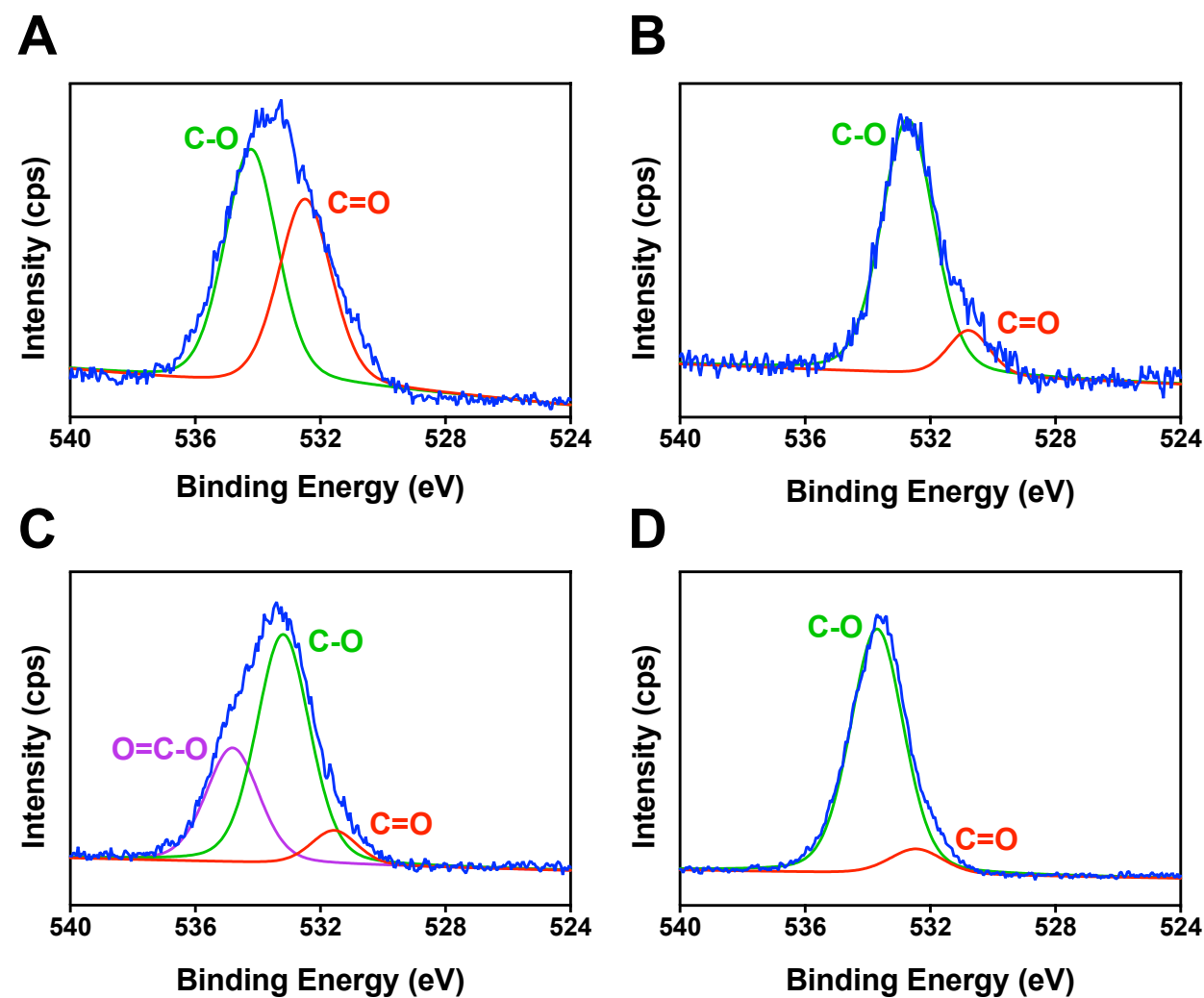

D
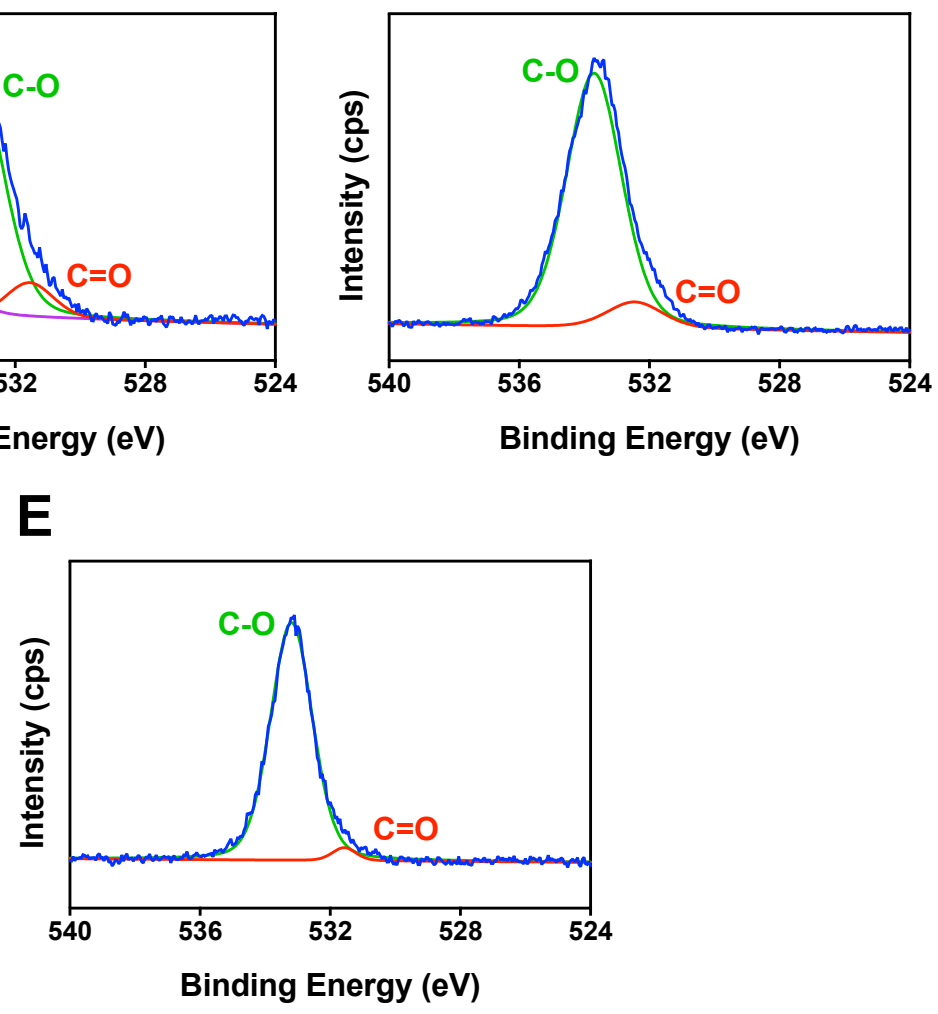

Figure S3. Deconvoluted XPS high-resolution spectra of O1s regions of (A) unmodified PDA, (B) PDA@PEG, (C) PDA@PEG-COOH, (D) PDA@PEG-NH ${ }_{2}$, and (E) PDA@PEG-Chol NPs with peak assignments inferred from literature. The increase in $\mathrm{C}-\mathrm{O}$ components observed in PEGylated NPs (B - E) compared to unmodified PDA NPs (A) is attributed to immobilization of $\mathrm{PEG}$ ligands on the surface of PDA NPs. The appearance of $\mathrm{O}=\mathrm{C}-\mathrm{O}$ components in (C) corresponds to the presence of PEG-COOH. 


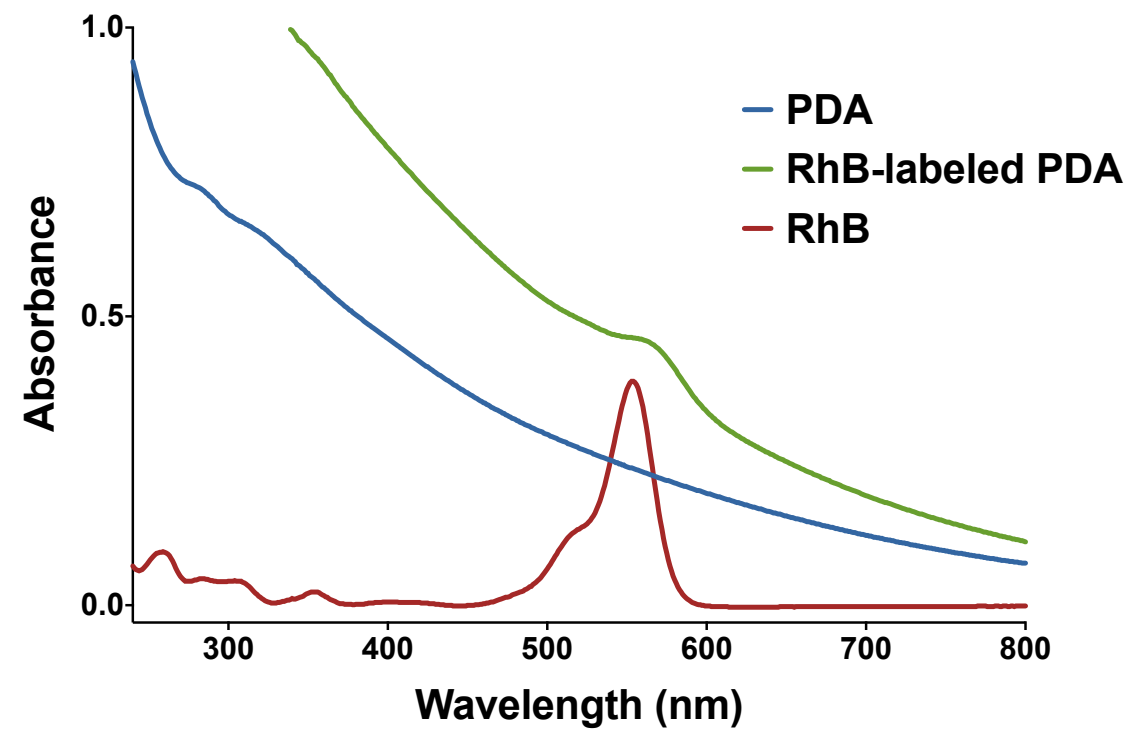

Figure S4. Representative UV-Vis spectra of unlabelled and RhB-labeled PDA NPs compared to $\mathrm{RhB}$ in ultrapure water. Even though PDA exhibits broad absorbance across the UV-Vis region, $\mathrm{RhB}$ peak is still distinguishable after labelling, appearing at $\lambda=560 \mathrm{~nm}\left(\mathrm{RhB} \lambda_{\max }=557 \mathrm{~nm}\right)$. 


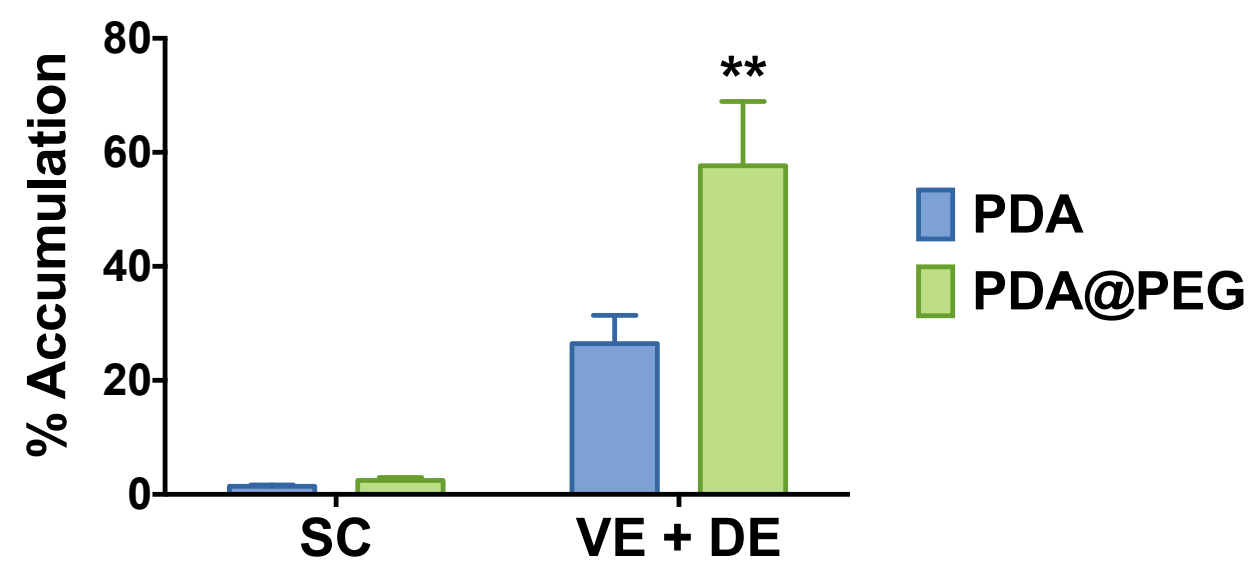

Figure S5. Results from a pilot ex vivo skin deposition study to determine distribution of RhBlabeled PDA and PDA@PEG NPs across skin compartments $(\mathrm{n}=3)$. Negligible fluorescence signals were obtained from tape-stripped stratum corneum (SC) and no signals were obtained from the receiver compartments, indicating no transdermal permeation and preferential accumulation of the NPs in the viable epidermis (VE) and dermis (DE) layers. Notably, PEGylated PDA NPs were able to achieve significantly higher intradermal deposition compared to unmodified PDA NPs $(p<0.01)$, which was attributed to their steric stability upon skin contact. 


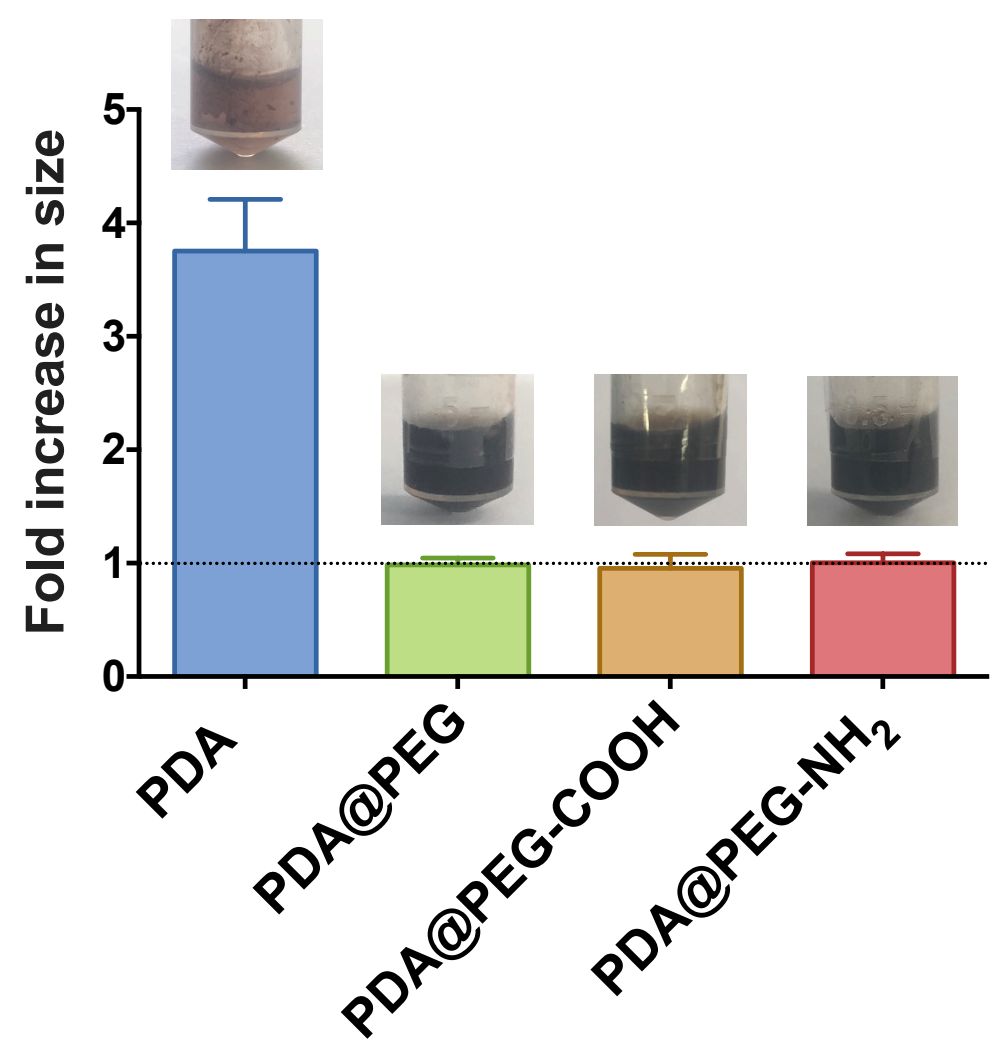

Figure S6. Change in particle size of unmodified and surface-modified PDA NPs upon incubation in skin-conditioned media for $24 \mathrm{~h}$. PDA NPs underwent severe aggregation leading to $\sim 4$ fold increase in size, most likely due to adsorption of skin-secreted proteins. On the other hand, NPs modified with various PEG ligands remained colloidally stable with a negligible change in particle size. 


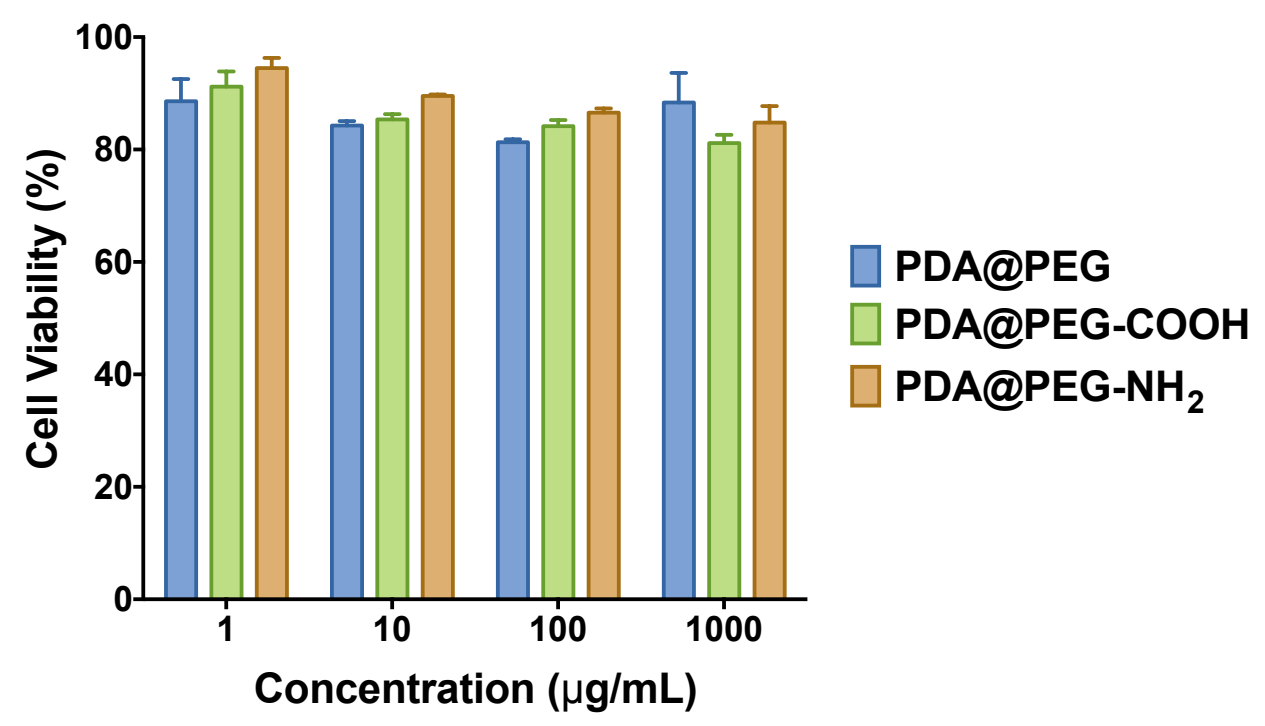

Figure S7. Cell viability of human dermal fibroblasts treated with various concentrations of PDA@PEG, PDA@PEG-COOH, and PDA@PEG-NH $\mathrm{NH}_{2}$ NPs in complete media for 72 h. 


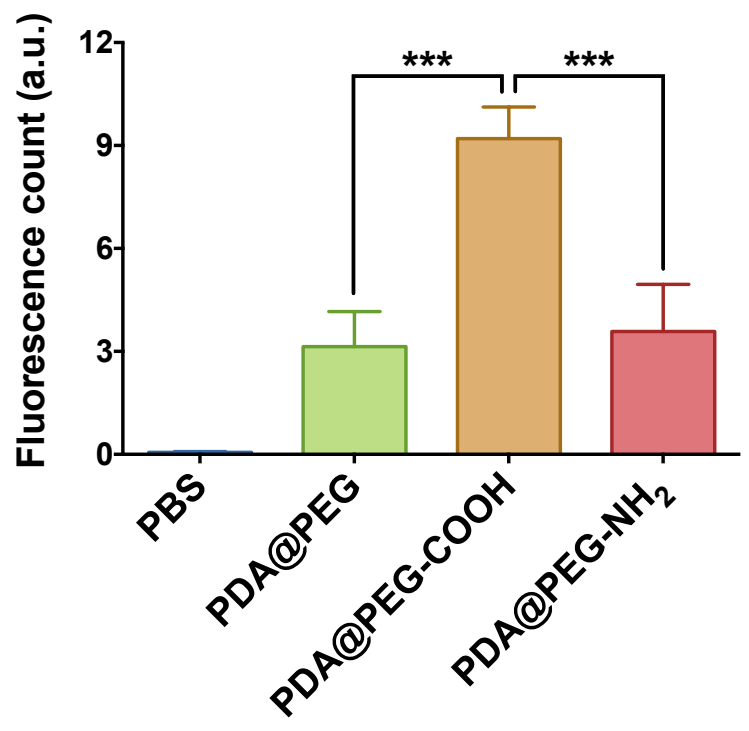

Figure S8. Quantified fluorescence signal intensities corresponding to the red fluorescence images of microtomed skin samples presented in Figure 6 of the main text. Significantly higher intensities were obtained from samples treated with RhB-labeled PDA@PEG-COOH NPs compared to PDA@PEG and PDA@PEG-NH $\mathrm{NH}_{2} \mathrm{NPs}$, consistent with the results in Figure 5B. Data are presented as mean $\pm \mathrm{SD}$ of pixel counts measured from regions of interest of equal area from three different images for each sample. ${ }^{* * *} p<0.001$ compared to PDA@PEG-COOH NPs. 


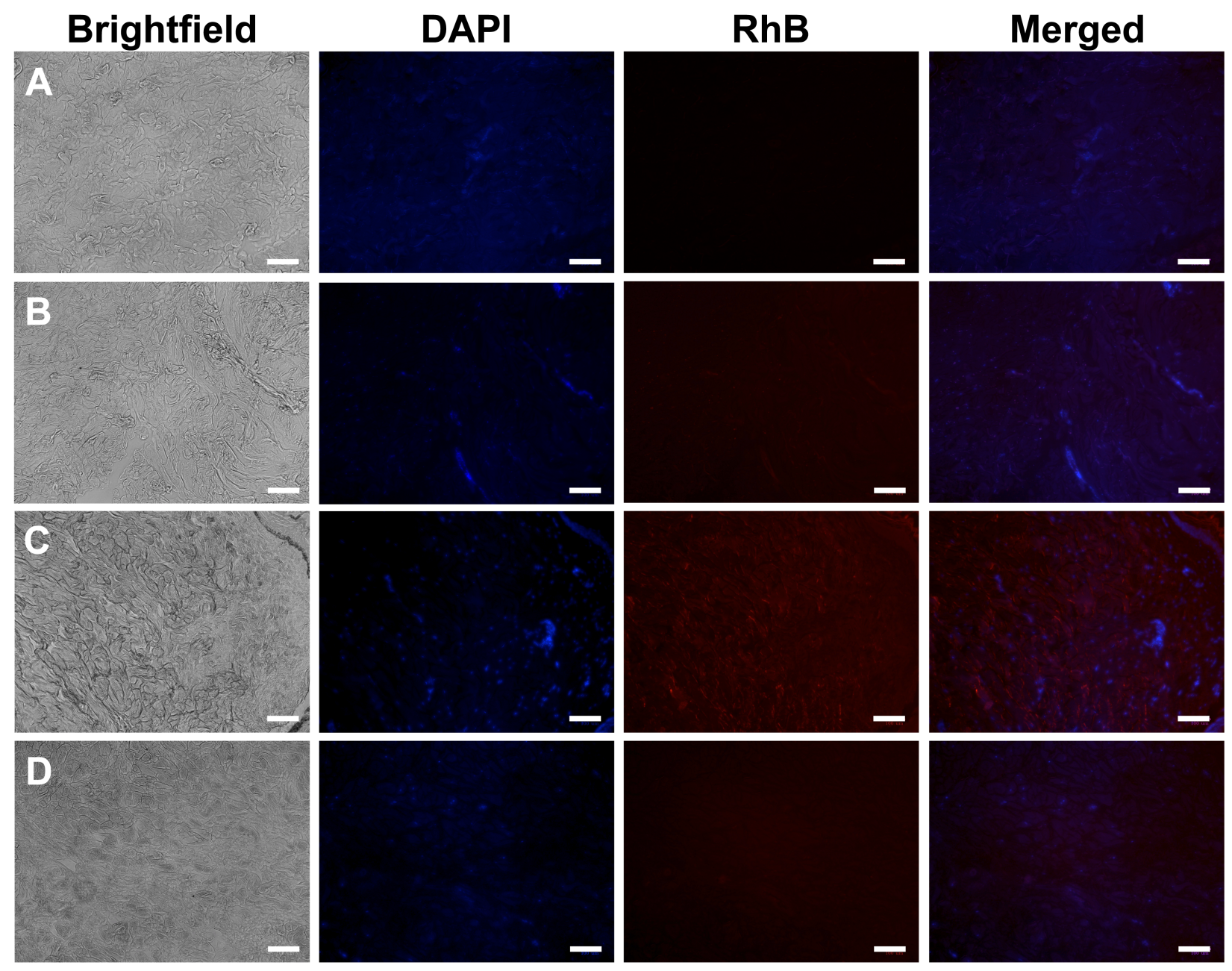

Figure S9. Representative brightfield and fluorescence images of the dermal regions of microtomed skin samples stained with DAPI after $24 \mathrm{~h}$ incubation with (A) PBS pH 7.4, (B) RhB-labeled PDA@PEG, (C) PDA@PEG-COOH, and (D) PDA@PEG-NH NPs; $_{2}$ Scale bar = $100 \mu \mathrm{m}$. Brighter red fluorescence signals were observed from the dermal layers of skin samples treated with PDA@PEG-COOH NPs (C) compared to PDA@PEG (B) and PDA@PEG-NH NPs (D), consistent with the results in Figure 5B of the main text. 


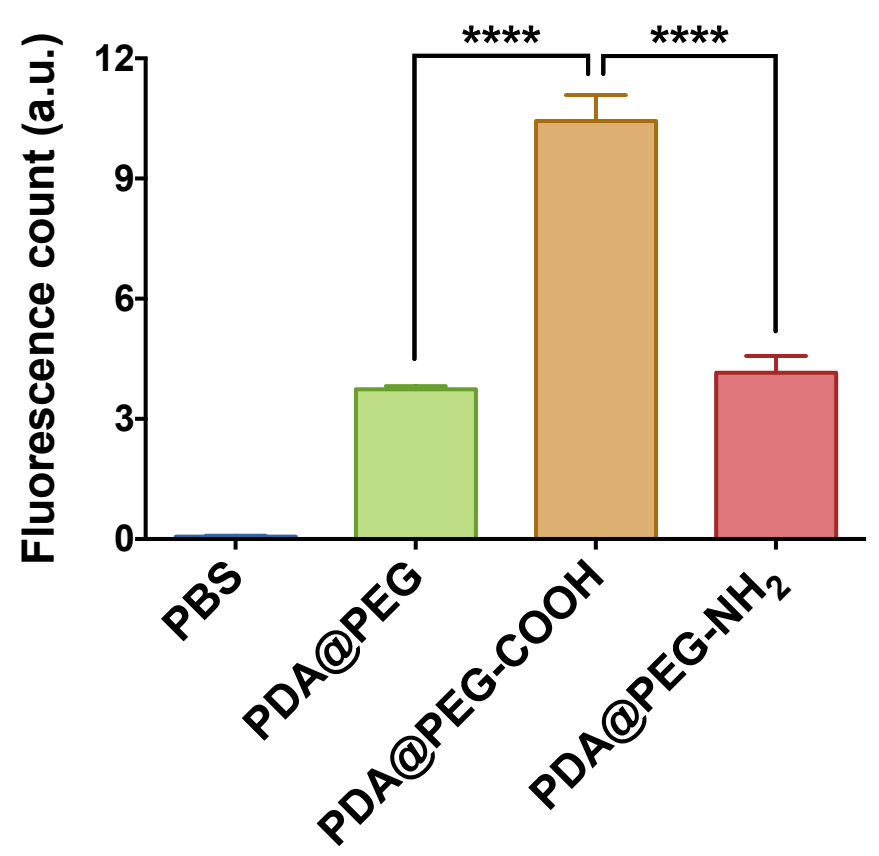

Figure S10. Quantified red fluorescence signal intensities of the dermal regions of microtomed skin samples presented in Figure S9. Significantly higher intensities were obtained from samples treated with RhB-labeled PDA@PEG-COOH NPs compared to PDA@PEG and PDA@PEG$\mathrm{NH}_{2}$ NPs, consistent with the results in Figure 5B of the main text. Data are presented as mean \pm $\mathrm{SD}$ of pixel counts measured from three different images for each sample. $* * * * p<0.0001$ compared to PDA@PEG-COOH NPs. 